\title{
Hard X-ray and radio observations of Abell 754
}

\author{
R. Fusco-Femiano ${ }^{1}$, M. Orlandini ${ }^{2}$, S. De Grandi ${ }^{3}$, S. Molendi ${ }^{4}$, L. Feretti ${ }^{5}$, G. Giovannini ${ }^{5,6}$, \\ M. Bacchi ${ }^{5,6}$, and F. Govoni ${ }^{5,6}$ \\ ${ }^{1}$ Istituto di Astrofisica Spaziale e Fisica Cosmica (IASF), CNR, via del Fosso del Cavaliere 100, 00133 Roma, Italy \\ e-mail: dario@rm.iasf.cnr.it \\ 2 IASF, CNR, Bologna, Italy \\ 3 Osservatorio Astronomico di Brera, Merate, Italy \\ ${ }^{4}$ IASF, CNR, Milano, Italy \\ 5 Istituto di Radioastronomia, CNR, Bologna, Italy \\ ${ }^{6}$ Dip. di Astronomia, Univ. di Bologna, Bologna, Italy
}

Received 2 July 2002 / Accepted 3 November 2002

\begin{abstract}
We present a long BeppoSAX observation of Abell 754 that reports a nonthermal excess with respect to the thermal emission at energies greater than $\sim 45 \mathrm{keV}$. A VLA radio observation at $1.4 \mathrm{GHz}$ definitely confirms the existence of diffuse radio emission in the central region of the cluster, previously suggested by images at 74 and $330 \mathrm{MHz}$ (Kassim et al. 2001), and reports additional features. Besides, our observation determines a steeper radio halo spectrum in the 330-1400 MHz frequency range with respect to the spectrum detected at lower frequencies, indicating the presence of a spectral cutoff. The presence of a radio halo in A754, considered the prototype of a merging cluster, reinforces the link between formation of Mpc-scale radio regions and very recent or current merger processes. The radio results combined with the hard X-ray excess detected by BeppoSAX give information on the origin of the electron population responsible for nonthermal phenomena in galaxy clusters. We discuss also the possibility that 26W20, a tailed radio galaxy with BL Lac characteristics located in the field of view of the PDS, could be responsible for the observed nonthermal hard X-ray emission.
\end{abstract}

Key words. galaxies: clusters: individual: A 754 - radio continuum: galaxies - X-rays: galaxies: clusters

\section{Introduction}

Shocks and turbulence associated with a major cluster merger event could provide the ingredients necessary to the formation of extended radio regions detected so far in a limited number of clusters, namely a diffuse magnetic field amplification and particle reacceleration (Tribble 1993; Roettiger et al. 1999a; Roettiger et al. 1999b; Brunetti et al. 2001). The existence of Mpc-scale radio halos or relics combined with the relatively short radiative lifetimes suggests an in-situ electron reacceleration induced by a very recent or current merger event. This hypothesis is supported by observational evidence (Feretti 1999). The existence of large radio regions could be at the origin of the nonthermal hard X-ray (HXR) emission detected in the Coma cluster (Fusco-Femiano et al. 1999; Rephaeli et al. 1999) and Abell 2256 (Fusco-Femiano et al. 2000) both showing extended radio emission. A considerable fraction of the input energy during a merger process can be released in particle acceleration and re-emitted in various energy bands. In particular, the same accelerated electrons responsible for the diffuse radio synchrotron emission can scatter the Cosmic Microwave Background (CMB) photons to produce nonthermal inverse

Send offprint requests to: $\mathrm{R}$. Fusco-Femiano,

e-mail: dario@rm.iasf.cnr.it
Compton (IC) X-ray emission. Alternative relativistic particles to primary re-accelerated electrons emitting in radio halos or relics and responsible for IC nonthermal HXR emission could be secondary electrons produced in the interactions of cosmic rays in the ICM (Dennison 1980; Blasi \& Colafrancesco 1999).

$\mathrm{X}$-ray and optical observations report a violent merger event in the galaxy cluster Abell 754 (Henry \& Briel 1995; Zabludoff \& Zaritsky 1995; Henriksen \& Markevitch 1996; Bliton et al. 1998; Markevitch et al. 1998; De Grandi \& Molendi 2001) and a numerical hydro/N-body model of this cluster (Roettiger et al. 1998) has shown that many of its observed morphological properties can be explained by a very recent merger $(<0.3 \mathrm{Gyr})$, slightly off-axis, between two clusters having a total mass-ratio less than 2.5:1. Therefore, the intracluster medium (ICM) of A754 appears to be a suitable place for the formation of radio halos or relics. As a consequence, radio and HXR observations of this cluster are relevant to establish robust evidence of the suggested link between the presence of nonthermal phenomena and merger activity in clusters of galaxies and to obtain information on the processes that can inject relativistic electrons. The cluster has been recently imaged at 74 and $330 \mathrm{MHz}$ with the NRAO VLA observatory (Kassim et al. 2001) suggesting the existence of a radio halo and at least one radio relic. Observations at higher resolution and 
sensitivity are required to confirm the obtained results. A754 was observed in hard X-rays with RXTE in order to search for a nonthermal component (Valinia et al. 1999). The fit to the PCA and HEXTE data set an upper limit of $\sim 1.4 \times 10^{-12} \mathrm{erg} \mathrm{cm}^{-2} \mathrm{~s}^{-1}$ in the $10-40 \mathrm{keV}$ band to the nonthermal emission.

In this paper we present the results of a high sensitivity VLA radio observation at $1.4 \mathrm{GHz}$ and of a long BeppoSAX observation, exploiting the unique capabilities of the PDS (Frontera et al. 1997) to search for HXR emission. We discuss the new radio data and the origin of the excess detected at energies above $\sim 45 \mathrm{keV}$ with respect to the thermal emission.

Throughout this paper, we assume a Hubble constant of $H_{0}=50 \mathrm{~km} \mathrm{~s}^{-1} \mathrm{Mpc}^{-1} h_{50}$ and $q_{0}=1 / 2$, so that an angular distance of $1^{\prime}$ corresponds to $86 \mathrm{kpc}\left(z_{\mathrm{A} 754}=0.054\right.$; Bird 1994). Quoted confidence intervals are at a $90 \%$ level, if not otherwise specified.

\section{Hard X-ray data}

\subsection{PDS and MECS data reduction}

The pointing coordinates of BeppoSAX are at $\mathbf{J} 2000 \alpha=$ $9^{\mathrm{h}} 9^{\mathrm{m}} 21.0^{\mathrm{s}}, \delta=-9^{\circ} 41^{\prime} 24.0^{\prime \prime}$. The total effective exposure time for the PDS was $\sim 84250 \mathrm{~s}$ in the two observations of 2000 May 6th and May 17th. The observed count rate was $0.333 \pm 0.021$ counts s$^{-1}$ in the $15-100 \mathrm{keV}$ energy range, at a confidence level of $\sim 16 \sigma$.

Since the source is rather faint in the PDS band (approximately 2 mcrab in the $15-100 \mathrm{keV}$ ), a careful check of the background subtraction must be performed. PDS spectra were extracted using the XAS v2.0 (Chiappetti \& Dal Fiume 1997). The background sampling was performed using the default rocking law of the two PDS collimators that samples $\mathrm{ON},+\mathrm{OFF}, \mathrm{ON},-\mathrm{OFF}$ fields for each collimator with a dwell time of $96 \mathrm{~s}$ (Frontera et al. 1997). When one collimator is pointing ON source, the other collimator is pointing toward one of the two OFF positions. We used the standard procedure to obtain PDS spectra (Dal Fiume et al. 1997); this procedure consists of extracting one accumulated background spectra in the two different $+/-$ OFF sky direction. The comparison between the two accumulated backgrounds ([+OFF] vs. $[-\mathrm{OFF}])$ shows a difference in the pointings for the first of the two observations. In particular, the background in the position $+\mathrm{OFF}$ was much greater than that in position -OFF. So, for the analysis, we have considered only the background in the -OFF position. In the second observation we do not find differences between the spectra extracted from the two offset positions. The background level of the PDS is the lowest and most stable obtained so far with the high-energy instruments on board satellites thanks to its equatorial orbit. No modelling of the time variation of the background is required. The correctness of the PDS background subtraction has been checked by verifying that the counts fluctuate at about zero flux as the signal falls below detectability. This happens at energies greater than $\sim 75 \mathrm{keV}$.

The total exposure time for the MECS was $\sim 1.85 \times 10^{5} \mathrm{~s}$ with a count rate of $0.748 \pm 0.002$ counts $^{-1}$. MECS data preparation and linearization were performed using the SAXDAS package under an FTOOLS environment. We have extracted an MECS spectrum from a circular region of $\sim 20^{\prime}$ radius (corresponding to about $1.7 \mathrm{Mpc}$ ). From the ROSAT PSPC radial profile, we estimate that about $95 \%$ of the total cluster emission falls within this radius. The background subtraction has been performed using spectra extracted from blank sky event files in the same region of the detector as the source. For more details see De Grandi \& Molendi (2001) that used the same MECS data to determine the temperature profile of A754.

A numerical relative normalization factor among the two instruments has been included in the fitting procedure (see next section) to account for (1) the fact that the MECS spectrum included emission out to $\sim 1.7 \mathrm{Mpc}$ from the X-ray peak, while the PDS field of view $\left(F W H M=1.3^{\circ}\right)$ covers the entire emission from the cluster; (2) the slight mismatch in the absolute calibration of the MECS and PDS response matrices employed; and (3) the vignetting in the PDS instrument (the MECS vignetting is included in the response matrix). The estimated normalization factor is $\sim 0.8$. In the fitting procedure, we allow this factor to vary within $15 \%$ from the above value to account for the uncertainty in this parameter.

\section{Radio data}

A radio observation at $1.365 \mathrm{GHz}$ was obtained with the Very Large Array (VLA) in D configuration on September 25, 2000, for a total integration time of about $2.5 \mathrm{~h}$. Data were calibrated and reduced with the Astronomical Image Processing System (AIPS), following the standard procedure (Fourier inversion, Clean and Restore, Self-calibration).

The radio image obtained with angular resolution of $70^{\prime \prime}$ is presented in Fig. 1, overimposed onto the optical image. The rms noise is of $0.1 \mathrm{mJy} / \mathrm{beam}$, i.e. about 5 times better than the NRAO VLA Sky Survey (NVSS) image (published by Giovannini et al. 1999). The diffuse emission detected here is complex and it is much more extended than in any previous image at any frequency.

The cluster halo is easily visible around the brightest optical $\mathrm{cD}$ galaxy. This emission is not detected in the NVSS, but is detected at $330 \mathrm{MHz}$ and $74 \mathrm{MHz}$ by Kassim et al. (2001). As pointed out by these authors, several discrete sources are embedded within the radio halo. In addition, we detect in the present image an extended diffuse emission to the east of the halo. Although a connection of this emission to the radio halo cannot be excluded, we tentatively classify this feature as a peripheral cluster relic. The cluster radio relic is detected by Kassim et al. (2001) at $74 \mathrm{MHz}$ and not at $330 \mathrm{MHz}$. At $74 \mathrm{MHz}$ it is much less extended than the feature detected here at $1.4 \mathrm{GHz}$, and it is located at the eastern boundary of our relic image. The possible connection relic-halo present in the $1.4 \mathrm{GHz}$ image does not appear in the $74 \mathrm{MHz}$ image.

The total flux density of the radio halo at $1.4 \mathrm{GHz}$, after subtraction of the discrete sources, is $86 \pm 2 \mathrm{mJy}$. Comparison to the total flux at $330 \mathrm{MHz}$ given by Kassim et al. (2001) leads to a spectral index $\alpha_{330}^{1400} \sim 1.5$. The halo spectrum appears to be steeper than that estimated by the previous observations in 


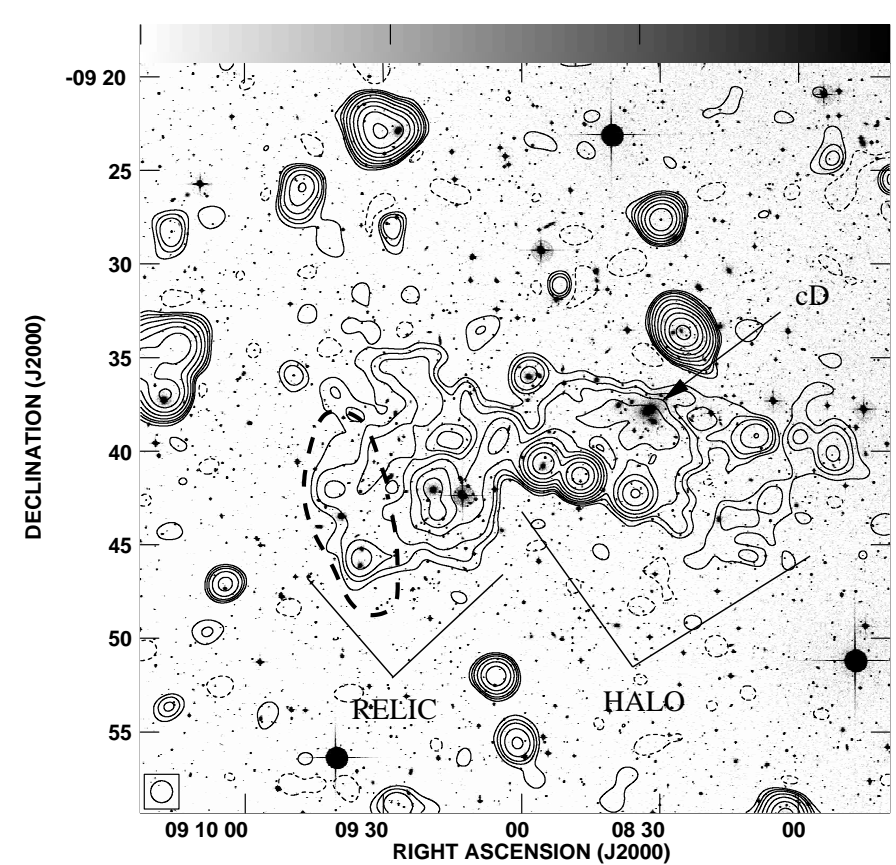

Fig. 1. Radio map at $20 \mathrm{~cm}$ with resolution of $70^{\prime \prime}$ (contours), overlayed onto the grey-scale image from the digitized ESO/SERC Southern Sky Survey. The $\sigma$ noise level in this map is $0.1 \mathrm{mJy} / \mathrm{beam}$. Contours are at $-0.3,0.3,0.6,1,2,4,8,16,32,64,128 \mathrm{mJy} / \mathrm{beam}$ The cluster $\mathrm{cD}$ galaxy is marked by an arrow. The cluster halo and relics are indicated. The bold dashed contour indicates the east relic of Kassim et al. (2001) at $74 \mathrm{MHz}$.

the $74-330 \mathrm{MHz}$ frequency band $\left(\alpha_{74}^{330} \sim 1.1\right)$, indicating the presence of a spectral cutoff. The total flux density of the relic is $69 \pm 2 \mathrm{mJy}$. Since the relic source in the map of Kassim et al. (2001) at $74 \mathrm{MHz}$ is significantly less extended than in the present map at $1.4 \mathrm{GHz}$, we obtain a lower limit $\sim 1$ for the spectral index in the range 74-1400 MHz. An upper limit of $\sim 1.7$ in the range $330-1400 \mathrm{MHz}$ is derived from the lack of detection in the $330 \mathrm{MHz}$ image of Kassim et al. (2001).

\subsection{Hard X-ray results}

The temperature profile derived from the MECS data analysis of this BeppoSAX observation confirms that A754 is an extremely disturbed and substructured cluster, as reported in previous X-ray and optical observations. The MECS spectrum is extracted from a circular region of radius $\sim 20^{\prime}$ that gives a mean temperature of $9.42_{-0.17}^{+0.16} \mathrm{keV}$ consistent with the value of $\sim 9 \mathrm{keV}$ determined from other X-ray instruments (ASCA GIS, Ginga LAC, RXTE PCA) within their respective errors bars. The iron abundance results to be $0.25_{-0.03}^{+0.04}$. These results are reported in De Grandi \& Molendi 2001.

To search for a nonthermal component in the spectrum of A754, we have performed a simultaneous fit (see Fig. 2) to the MECS and PDS data with a MEKAL model that reports an average gas temperature of $\sim 9.4 \mathrm{keV}\left(\chi^{2}=194.4\right.$ for 158 d.o.f.). A systematic error of $1 \%$ was added in quadrature to the statistical uncertainties of the spectral data of the PDS, on the basis of the results obtained from routinely performed Crab Nebula observations. The normalization factor for the two data sets is

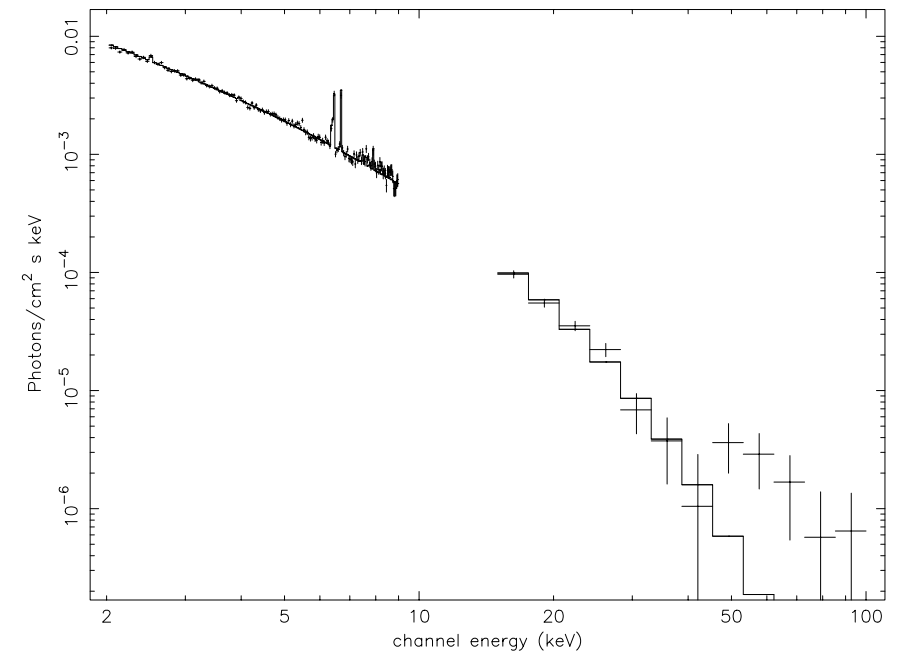

Fig. 2. MECS data in the $2-9 \mathrm{keV}$ energy range and PDS data at energies above $15 \mathrm{keV}$ of the BeppoSAX observation of A754. The continuous line is the fit with a MEKAL model. The derived mean gas temperature is of $\sim 9.4 \mathrm{KeV}$ within a region of $20^{\prime}(1.7 \mathrm{Mpc})$ of radius.

$\sim 0.72$. At energies above $\sim 45 \mathrm{keV}$ there is a conspicuous excess with respect to thermal emission at a confidence level of $\sim 3.2 \sigma$. This long BeppoSAX observation is composed of two observations with a time interval of few days and the separate data analysis does not evidence significative variability. We infer a robust indication for the nonthermal nature of the detected excess by fitting the MECS/PDS data with a 2-T model and with a $\mathrm{T}$ plus a power law model. In the first model one of the temperatures has the value of $\sim 9.4 \mathrm{keV}$, while the second temperature assumes unrealistic values greater than $50-60 \mathrm{keV}$ that strongly support a nonthermal mechanism for the second component. In the second model the fit, with a $\chi^{2}$ value of 177.4 for 156 d.o.f., is not able to determine the spectral index, but the improvement of the $\chi^{2}$ value with respect to a single thermal component (MEKAL model) is significant at more than $99.92 \%$ confidence level, according to the F-test. In the 10-40 keV energy range the fit gives a nonthermal flux of $\sim 1.6 \times 10^{-12} \mathrm{erg} \mathrm{cm}^{-2} \mathrm{~s}^{-1}$ consistent with the upper limit reported by the RXTE observation (Valinia et al. 1999).

In the next session we discuss the origin for the detected nonthermal excess considering the presence of the radio halo in the ICM of the cluster and that of the radio galaxy 26W20, which displays BL Lacertae characteristics (Silverman et al. 1998), in the field of view of the PDS at a distance of about $27^{\prime}$ from the BeppoSAX pointing.

\section{Discussion}

Nonthermal X-ray emission has been detected in the Coma cluster and Abell 2256 by the PDS onboard BeppoSAX, while a flux upper limit has been reported for Abell 3667 (FuscoFemiano et al. 2001). The deviation of these "hard tails" from the the thermal bremsstrahlung spectra appears to start at energies above $20-25 \mathrm{keV}$. A likely origin for the detected nonthermal HXR radiation is IC emission by scattering of CMB photons by the radio synchrotron electrons responsible for extended radio regions present in the center (radio halo) or in the 
periphery (radio relic) of the cluster. Alternative mechanisms to the IC model have been proposed (Blasi \& Colafracesco 1999; Sarazin \& Kempner 2000; Dogiel 2000; Blasi 2000; Liang et al. 2002), some of these motivated by the discrepancy between the value for the intracluster magnetic field derived by the BeppoSAX observation of the Coma cluster $\left(B_{X} \sim 0.16 \mu \mathrm{G}\right.$; Fusco-Femiano et al. 1999) and the value derived from the Faraday rotation $\left(B_{\mathrm{FR}} \sim 6 \mu \mathrm{G}\right.$; Feretti et al. 1995) of polarized radiation of sources through the ICM. Recently, Newman et al. (2002) have pointed out that many and large uncertainties are associated to the determination of $B_{\mathrm{FR}}$ (see also Govoni et al. 2002). However, this discrepancy can be attenuated considering models that include the effects of more realistic electron spectra, spatial profiles of the magnetic fields and anisotropies in the pitch angle distribution of the electrons (Goldshmidt \& Rephaeli 1993; Brunetti et al. 2001; Petrosian 2001). Another possibility is that the detected excesses are due to the presence of very obscured sources like Circinus (Matt et al. 1999) in the field of view of the PDS $\left(1.3^{\circ}\right)$. In the case of Coma and A2256 the analysis of the MECS images excludes the presence of this kind of sources in the central region $\left(\sim 30^{\prime}\right.$ in radius) of the cluster unless the obscured source is within $2^{\prime}$ of the central bright cores. We have re-observed A2256 after about two years from the first observation and the two spectra are consistent (Fusco-Femiano et al. 2002). Besides, both observations comprise two exposures with a time interval of $\sim 1$ year and $\sim 1$ month, respectively and all these observations do not show significant flux variations. These results and the fact that the two clusters, Coma and A2256, with a detected HXR excess both have extended radio regions strongly supports the idea that a diffuse nonthermal mechanism involving the ICM is responsible for the observed excesses. Support to this interpretation is given by a second recent RXTE observation of the Coma cluster (Rephaeli \& Gruber 2002) that confirms the previous observation (Rephaeli et al. 1999) of a likely presence of a nonthermal component in the spectrum of the cluster.

For A754 we discuss two possible origins for the detected excess at the level of $\sim 3.2 \sigma$, lower than that measured in Coma and A2256 $(\sim 4.5 \sigma)$. As reported in the Introduction, the ICM of A754 appears to be a suitable place to host relativistic electrons considering that X-ray and optical observations indicate that the cluster is undergoing a violent merger process, and that Hydro/N-body simulations show that the merger must be very recent. The time elapsed from the start of the merger in A754 $(<0.3 \mathrm{Gyr})$ is not greater than the radiative lifetime for electrons with $\gamma \sim 10^{4}$. Shocks and turbulence associated with a recent or current major merger event can be able to provide energy for particle acceleration and magnetic field amplification to produce diffuse radio emission (Tribble 1993; Brunetti et al. 2001). This scenario seems to be confirmed by the presence of diffuse radio emission (Kassim et al. 2001) and now definitely confirmed by our deeper VLA observation at higher resolution at $1.4 \mathrm{GHz}$. The nonthermal HXR detected by the PDS could be IC emission by the same radio electrons scattering CMB photons. The lack of variability in the nonthermal flux detected by this long BeppoSAX observation is compatible with a diffuse mechanism of the observed excess. Assuming that the measured nonthermal flux of $\sim 10^{-11} \mathrm{erg} \mathrm{cm}^{-2} \mathrm{~s}^{-1}$ in the 40-100 keV energy range is due to IC emission, the volume-averaged intracluster magnetic field is $\sim 0.1 \mu \mathrm{G}$ obtained combining the radio halo and X-ray fluxes (see FuscoFemiano et al. 1999, Eq. (1)) and using our measured value of 1.5 for the radio halo index. A similar value of $B_{X}$ is obtained for the radio relic assuming a radio spectral index of 1.7. The equipartition value of the magnetic field, computed with standard assumptions, is in the range $0.3-0.6 \mu \mathrm{G}$.

The presence of diffuse radio radiation in A754, considered the prototype of a merging cluster, is a further evidence of the link between Mpc-scale radio emission and very recent or current merger processes (Feretti 1999). Radio observations and the detection of nonthermal HXR emission are of great importance for the understanding of the origin of the electron population responsible for nonthermal phenomena in clusters of galaxies. In the Coma cluster, the prototype of halo clusters, the spectral cut-off (Deiss et al. 1997) represents a strong indication for the presence of a cut-off in the emitting electron spectrum. This cut-off and the HXR excess detected in the Coma cluster may be naturally accounted for in the context of re-acceleration models (Brunetti et al. 2001) in which relativistic electrons with $\gamma \sim 100-300$, produced by several sources in galaxy clusters and accumulated in the cluster volume over cosmological time, can be re-accelerated by various processes at the energies required to explain the radio halo in the Coma cluster. This cut-off is not naturally expected if the radio emission is produced by secondary electrons (Brunetti 2002) due to the decay of charged pions generated in cosmic ray collisions (Dennison 1980; Blasi \& Colafrancesco 1999; Dolag \& Ensslin 2000; Miniati et al. 2001). The radio and HXR observations of A754 seem to confirm the scenario described by the re-acceleration models in the Coma cluster. The detection of the hard excess in A754 determines, in the framework of the IC model, a volume-averaged intracluster magnetic field of the same order $\left(B_{\mathrm{X}} \sim 0.1 \mu \mathrm{G}\right)$ of that determined in the Coma cluster (Fusco-Femiano et al. 1999). This value of $B_{\mathrm{X}}$ implies relativistic electrons at energies $\gamma \sim 10^{4}$ to explain the observed diffuse synchrotron emission. At these energies IC losses may determine a cut-off in the electron spectrum in agreement with the spectral cut-off observed in A754. So, the radio and HXR results obtained by our observations of A754 support the scenario that primary and not secondary electrons are responsible for nonthermal emission in clusters of galaxies.

However, we cannot ignore the presence of the radio galaxy $26 \mathrm{~W} 20$ in the field of view of the PDS, located at a distance of about $27^{\prime}$ from the BeppoSAX pointing, that could be responsible for the hard excess in A754. This source, discovered in the Westerbork radio survey by Harris et al. (1980), shows a $\mathrm{X}$-ray bright core similar to that of a BL Lac object but with weak emission lines and this makes 26W20 different from a typical BL Lac object. The source is preferred to be classified as tailed radio galaxy for the presence of a single jet or tail and being member of a small group of galaxies. The radio galaxy has had several X-ray observations due to its close proximity to A754. It was first observed with Einstein (Harris et al. 1984) and the IPC spectrum could be equally well fit by a power law (energy index $0.8 \pm 0.4$ ) or with a thermal model $(k T \sim 3 \mathrm{keV})$. 


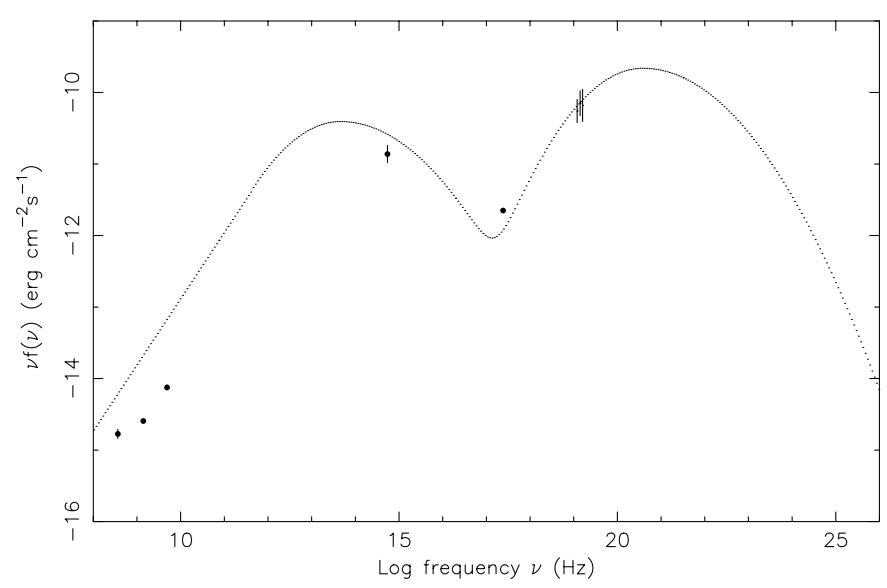

Fig. 3. Spectral energy distribution for $26 \mathrm{~W} 20$. The first and third radio points are taken from NED, the second from NVSS. The optical point from the USNO catalog and the ROSAT point from the PSPC observation by Silverman et al. (1998). The highest energy points refer to the hard excess detected by the PDS observation. The dotted line is the fit to the SED with a SSC model.

In the following the nonthermal nature of the X-ray emission was clarified by a ROSAT observation. In particular, the fit to an on-axis PSPC observation gives a core emission described by a nonthermal model with energy index $1.32 \pm 0.17$ and $\log N_{\mathrm{H}}=20.90 \pm 0.04$. The $\mathrm{X}$-ray luminosity is $\sim 3 \times$ $10^{43} \mathrm{erg} \mathrm{s}^{-1}$ in the $0.5-3 \mathrm{KeV}$ energy range (Silverman et al. 1998). The source shows variability. An $18 \%$ increase in the luminosity has been determined by two PSPC observations within 5 days in 1992.

The spectral energy distributions (SED) of BL Lac sources are characterized by two main peaks. The peak at low energy is commonly explained as synchrotron emission by relativistic electrons while that at higher energies as IC emission of the same electrons scattering the synchrotron photons (e.g. Dermer et al. 1997; Ghisellini et al. 1998).

In Fig. 3 we show the SED for 26W20 where the highest energy points refer to the PDS observation assuming that this source is responsible for the detected excess. The fit with a Synchrotron Self-Compton (SSC) model is consistent with the SED of a BL Lac object (Perry 2001; Padovani et al. 2002; Giommi et al. 2002), but a flat energy index of about 0.3 is required to extrapolate the flux detected by ROSAT in the PDS energy range, taking into account the angular response of the detector. The inclusion of the PDS points makes difficult to fit well all the points of the SED. However, due to the variability of this kind of sources only simultaneous observations can be used to fix the spectral properties in a large energy band. Unfortunately, the source does not appear in the field of view of the MECS because hidden by one of the calibration sources of the instrument.

\section{Conclusions}

Our VLA observation at $1.4 \mathrm{GHz}$ definitely confirms diffuse radio emission in $\mathrm{A} 754$ composed by a radio halo and a relic, although a connection relic-halo cannot be excluded. The relic image at $1.4 \mathrm{GHz}$ appears much more extended than that detected at $74 \mathrm{MHz}$ by Kassim et al. (2001). Our observation determines with accuracy the slope of the radio halo spectrum in the $0.3-1.4 \mathrm{GHz}$ frequency range that results to be steeper than that obtained in the band 74-330 MHz, indicating the presence of a radio spectral cutoff. A BeppoSAX observation of A754 shows a nonthermal HXR excess at energies above $\sim 45 \mathrm{keV}$ with respect to the thermal emission, at a confidence level of $\sim 3 \sigma$. One possible explanation is IC emission for the same relativistic electrons responsible for the diffuse radio emission. This interpretation has been invoked to explain the nonthermal emission detected in Coma and A2256 both showing extended radio regions. The lack of significative variability of the nonthermal flux detected in A754 is consistent with a diffuse mechanism for the observed excess. This detection, that needs to be confirmed by future observations, and the radio results seem indicate that primary re-accelerated electrons and not secondary electrons are at the origin of the observed nonthermal emission in clusters of galaxies. At present, it exists also the possibility that the radio galaxy 26W20, located in the field of view of the PDS, is responsible for the reported hard excess. The extrapolation of the ROSAT flux in the PDS energy range requires a flat index to explain the nonthermal HXR flux. However, considering the non simultaneity of the ROSAT and BeppoSAX observations the SED may have assumed a different shape at the moment of the BeppoSAX observation. To discriminate between these two interpretations a hard X-ray observation with imaging instruments is necessary.

Acknowledgements. We thank G. Brunetti and P. Giommi for useful suggestions regarding the interpretation of the results. We are grateful to the referee for the stimulating comments.

\section{References}

Bird, C. M. 1994, AJ, 107, 1637

Blasi, P. 2000, ApJ, 525, 603

Blasi, P., \& Colafrancesco, S. 1999, Astropart. Phys., 12, 169

Bliton, M., Rizza, E., Burns, J. O., Owen, F. N., \& Ledlow, M. J. 1998, MNRAS, 301, 609

Brunetti, G., Setti, G., Feretti, L., \& Giovannini, G. 2001, MNRAS, 320,365

Brunetti, G. 2002, invited review in the Meeting: Matter and Energy in Clusters of Galaxies, ASP Conf. Ser., ed. S. Bowyer, \& C.-Y. Hwang [astro-ph/0208074]

Chiappetti, L., \& Dal Fiume, D. 1997, in Data Analysis in Astronomy, ed. V. Di Gesu' et al. (Singapore: World Scientific), 101

Dal Fiume, D., Frontera, F., Nicastro, L., et al. 1997, in Data Analysis in Astronomy, ed. V. Di Gesu' et al. (Singapore: World Scientific), 111

De Grandi, S., \& Molendi, S. 2001, ApJ, 551, 153

Dennison, B. 1980, ApJ, 239, 93

Dermer, C. D., Sturner, S. J., \& Schlickeiser, R. 1997, ApJS, 109, 103

Deiss, B. M., Reich, W., Lesch, H., \& Wielebinski, R. 1997, A\&A, 321, 55

Dennison, B. 1980, ApJ, 239, L93

Dogiel, V. A. 2000, A\&A, 357, 66

Dolag, K., \& Ensslin, T. A. 2000, A\&A, 362, 151

Dressler, A. 1980, ApJS, 42, 565

Feretti, L., Dallacasa, D., Giovannini, G., \& Tagliani, A. 1995, A\&A, 302,680 
Feretti, L. 1999, in Diffuse Thermal and Relativistic Plasma in Galaxy Clusters, ed. H. Bohringer, L. Feretti, \& P. Schuecker (MPE Rep. 271; Garching: MPE), 3

Frontera, F., Costa, E., Dal Fiume, D., et al. 1997, A\&AS, 122, 357

Fusco-Femiano, R., Dal Fiume, D., Feretti, L., et al. 1999, ApJ, 513, L21

Fusco-Femiano, R., Dal Fiume, D., De Grandi, S., Feretti, L., et al. 2000, ApJ, 534, L10

Fusco-Femiano, R., Dal Fiume, D., Orlandini, M., et al. 2001, ApJ, 552, L100

Fusco-Femiano, R., et al. 2002, to appear in The Proc. of Workshop "Matter and Energy in Clusters of Galaxies", April 2002, Taiwan, ed. S. Bowyer, \& C.-Y. Hwang, ASP Conf. Ser. [astro-ph/0207241]

Ghisellini, G., Celotti, A., Fossati, G., Maraschi, L., \& Comastri, A. 1998, MNRAS, 301, 451

Giommi, P., Massaro, E., \& Palumbo, G. 2002, Proc. of Workshop "Blazar Astrophysics with BeppoSAX and Other Observatories", December 10-11, 2001, Frascati, Italy, in press

Giovannini, G., Feretti, L., Venturi, T., Kim, K. T., \& Kronberg, P. P. 1993, ApJ, 406, 399

Giovannini, G., Tordi, M., \& Feretti, L. 1999, New Astron., 4, 141

Govoni, F., Feretti, L., Giovannini, G., et al. 2002, to appear in the Proc. of Matter and Energy in Clusters of Galaxies, 23-27 April, Chung-Li, Taiwan, ed. S. Bowyer \& C.-Y. Hwang.

Goldshmith, O., \& Rephaeli, Y. 1993, ApJ, 411, 518

Harris, D. E., Pineda, F. J., Delvaille, J. P., et al. 1980, A\&A, 90, 283
Harris, D. E., Costain, C. H., \& Dewdney, P. E. 1984, ApJ, 280, 532

Henriksen, M. J., \& Markevitch, M. L. 1996, ApJ, 466, L79

Henry, J. P., \& Briel, U. G. 1995, ApJ, 443, L9

Kassim, N. E., Clarke, T. E., Enßlin, T. A., Cohen, A. S., \& Neumann, D. M. 2001, ApJ, 559, 785

Liang, H., Dogiel, V. A., \& Birkinshaw, M. 2002, MNRAS, in press [astro-ph/0208509]

Markevitch, M., Forman, W. R., Sarazin, C. L.

Matt, G., Guainazzi, M., Maiolino, R., et al. 1999, A\&A, 341, L39

Miniati, F., Jones, T. W., Kang, H., \& Ryu, D. 2001, ApJ, 562, 233

Newman, W. I., Newman, A. L., \& Rephaeli, Y. 2002 [astro-ph/0204451]

Padovani, P., Perlman, E. S., \& Landt, H. 2002, in preparation

Perry, M. 2001, Ph.D. Thesis

Petrosian, V. 2001, ApJ, 557, 560

Rephaeli, Y., Gruber, D. E., \& Blanco, P. 1999, ApJ, 511, L21

Rephaeli, Y., \& Gruber, D. 2002 [astro-ph/0207443]

Roettiger, K., Stone, J. M., \& Mushotzky, R. F. 1998, ApJ, 493, 62

Roettiger, K., Stone, J. M., \& Burns, J. O. 1999, ApJ, 518, 594

Roettiger, K., Burns, J. O., \& Stone, J. M. 1999, ApJ, 518, 603

Sarazin, C. L., \& Kempner, J. C. 2000, ApJ, 533, 73

Sijbring, L.G. 1993, Ph.D. Thesis, Groeningen Univ.

Silverman, J. D., Harris, D. E., \& Junor, W. 1998, A\&A, 335, 443

Tribble, P. C. 1993, MNRAS, 261, 57

Valinia, A., Henriksen, M. J., Loewenstein, M., et al. 1999, ApJ, 515, 42

Zabludoff, A. I., \& Zaritsky, D. 1995, ApJ, 447, L21 